\title{
Subject Identifier with Attribute Identifier
}

National Cancer Institute

\section{Source}

National Cancer Institute. Subject Identifier with Attribute Identifier. NCI Thesaurus. Code C94159.

A unique symbol that establishes identity of the subject. 